\title{
Multi-Level Indoor Navigation Ontology for High Assurance Location-Based Services
}

\author{
Sanya Khruahong*, Xiaoying Kong, Kumbesan \\ Sandrasegaran \\ Centre for Real-time Information Networks, \\ University of Technology Sydney \\ Sydney, Australia
}

\author{
Li Liu \\ Faculty of Engineering and Information Technologies \\ The University of Sydney \\ Sydney, Australia
}

\begin{abstract}
Indoor navigation will become an important application on a smartphone for Location-Based Service (LBS). An indoor navigation system should work under normal circumstances and during emergencies, such as fires, during a building power shut down, alarm, etc. The LBS should be able to help users find the best exit route to the outside of the building under all circumstances and with high reliability. In this research, we develop an indoor ontology model for indoor navigation. This ontology model defines the indoor environment attributes such as location nodes, and connection points. The location nodes with the location information allow navigation in the indoor environment. Connection points are able to separate the map zones and the building floors into a "Map sheet." This ontology approach allows the LBS works in both normal circumstances and emergencies. This model provides a reliable indoor navigation system for LBS.
\end{abstract}

Keywords—indoor navigation; location-based service; ontology

\section{INTRODUCTION}

New technologies are integrating physical, digital and human systems in to high assurance modern smart city system [1]. The smartphone is one such smart city technology for the Internet of Things (IoT). People use GPS receiver on their smartphones for navigation while travelling. However, travelling is not restricted to just the outdoors; there is also a requirement for indoor navigation for which GPS may not be effective. Indeed $90 \%$ of human activities occur in an indoor environment [2]. Multiple technologies including $\mathrm{Wi}-\mathrm{Fi}$, Bluetooth, and RFID are currently used for indoor navigation. Support for these technologies requires the accurate information provided by a digital map to help users find their correct position with required accuracy. Indoor navigation is one of the functions for LBS with high reliability, one of its quality requirements. As well as working under normal circumstances, the LBS should still work in emergencies, such as fires, building power shut down, etc. The high assurance LBS should be able to help users find the best exit route to the outside of a multiple level office building, shopping mall, etc. under all circumstances with high reliability. Emergency Rescue Localization (ERL) systems use Wireless LAN (WLAN) and cameras to find the location information inside a building [3]. However, these systems require building power, which may not be available during emergencies. Therefore, research in indoor way-finding needs to focus more on complex situations in complex environments. Multi-level indoor information is thus crucial for the development of indoor navigation applications to enable the user to link their current position to other floors of the building and navigate between them.

This research presents an Indoor Localization Ontology Model for Location Based Services (LBS) using a high assurance indoor navigation model. We design the Ontology model using the digital map of each floor linked together with multi-level node techniques. The ontology is designed to describe the nodes for indoor navigation search under both normal and emergency situations. The model is designed to provide indoor localization information, which is compatible with the combination of $\mathrm{Wi}-\mathrm{Fi}$, magnetic and Bluetooth devices to determine the user's current position. This ontology model contributes to a new standard for indoor localization information for LBS indoor navigation.

This paper is organized as follows. Section II reviews related work. Section III presents the indoor navigation ontology model. Section IV applies the ontology model to LBS. Section V draws the conclusion and indicates future directions.

\section{RELATED WORK}

Indoor navigation needs three parts: location-sensing technologies, the location knowledge of indoor environment, and the user route selections for navigation.

Location-sensing technologies are essential technologies for tagging, tracking, locating and monitoring and producing a huge repository of data [1]. A Location-Based Service (LBS) uses the current position of the mobile device obtained by using various devices and technologies [4-8] for navigation. Currently there are no standards for indoor navigation. Modern smartphones now embed some sensors such as accelerometers, magnetometers, light, ultrasonic, Bluetooth etc., which allow the development of mobile applications for location determination. In LBS, location measurements from sensors are meaningful only when they relate to the surrounding indoor environment. Ontology is a tool for representing the knowledge of indoor location environment.

Ontology is a set of naming and description of the types, properties, and interrelationships of the entities. Ontology has the structure of a dynamic database, which is efficient for LBS searching development [9]. The ontology cab be developed 
using Web Ontology Language (OWL) [10]. In literature, ontology techniques have led to be an ontology framework for indoor navigation systems such as indoorGML and BIGML $[11,12]$, which focus on the representation of indoor spaces.

Wang et al. designed an ontology for representing a semantic location in indoor navigation models [13]. This ontology shows the semantic information and multilayer semantic location, which consists of two types of information: static information such as the structure of the room, and dynamic information such as the sensing areas. However, this ontology is limited for presenting a map only on one floor, not linked to the other floors in multi-floor buildings. A hybrid modeling technique is proposed in OntoNav[14] by modeling geometric and semantic information for user navigation. The ontology OntoNav addresses the linking of other floors in the building; users need to select the path rule for traveling, and the restriction of the route may be hard for maintenance. A colour Petri Net model (CPN) using an RDF format for ontology has been developed for an indoor location-based system [15]. This model is able to identify the properties of core classes (such as subject, predicate, and object onto places), and map these properties onto CPN places. This approach may need to develop with OWL ontology. Scholz, J. and Schabus, S. adopted an ontology to support autonomous indoor navigation in the production environment [16]. This research used RFID and ultrasound technology to support autonomous navigation in the indoor space and develop a tracking system called LotTrack. However, the installation of RFID in buildings will be a large investment. Another approach for an indoor location used a Genetic Algorithm (GA) and a neural network [17] to collect positional data using RFID tags, RSS information, and four reader devices. This research was limited in scope, covering only one level.

From the literature, we found that previously ontology design has not been used for linking vertical and horizontally inside a multi-level building using indoor localization information. No emergency situation is considered in ontology design. To resolve these problems, in this paper we present an ontology design for multi-level semantic indoor location navigation.

\section{MULTI-LEVEL INDOOR NAVIGATION ONTOLOGY MODEL}

In this section, we will present our indoor navigation ontology model. We start from LBS navigation process for users first and then present out ontology design at architecture and detailed levels.

\section{A. LBS Indoor Navigation Process For Users}

Considering the usability of a LBS indoor navigation, we applied the industry practice using three-step navigation process flow for users [7]: (a) Indoor Positioning, (b) Geofencing, and (c) Way-finding.

Indoor positioning - Calculating the user's current position and display the current position on a smartphone as a "Blue dot” on a digital map.

Geo fencing - Defining a virtual boundary around a real geographical area. A radius of interest is established that can trigger an action in a geo-enabled smartphone. The names of the places within the radius are visible to the users.

Way-finding - Finding the optimal route to a destination of interest, and displaying the direction to the destination of interest. In our design, we propose regular routes and emergency routes for high assurance LBS.

To fit this user navigation process, we design the ontology to provide indoor location information.

\section{B. Architectural Design of the Ontology}

The architecture of the Multi-Level Indoor Navigation Ontology is designed using a UML diagram as Figure 1.

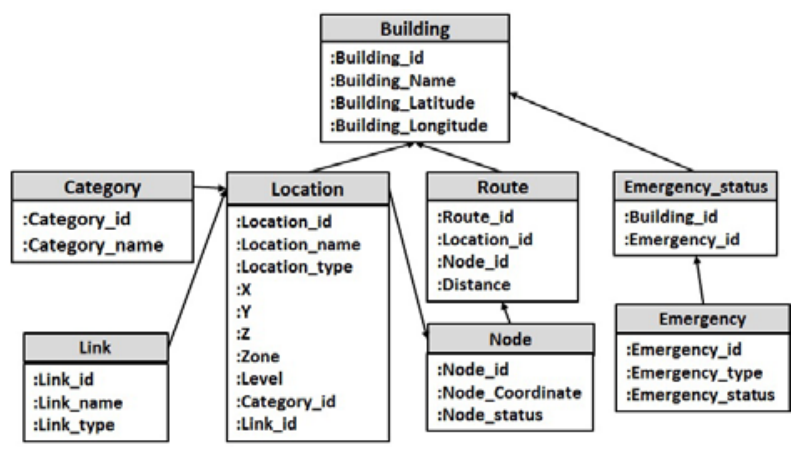

Fig. 1. Multi-Level Indoor Navigation Ontology

The ontology is desigend using classes and associated attributes. The major classes are: "Building", "Location", "Route”, "Link”, "Emergency”, "Emergency_Status”. The major classes are desigend as follows.

Building: Building is the main class for user navigation. The attributes of "Building" include: building ID, building name, the number of floor of the building, building latitude and building longitude.

Location: This is the class to define the physical location of a thing. In our ontology, we use 'node' to define three types of location: Place Location node (Place_Location), user mobile location node (Mobile_Location), and User Device Location node (Device_Location). The details of the location types and nodes will be described in the next section.

Node: Node is the class to define the inforamtion of the place. Node has attributes of Node ID, Node coordinate, Place name, Category for user searching service type criteria, Zone in the floor, Level in the building, and node Status. These atributes will be defined in Table 1. Figure 2 shows the physical nodes and links in a building.

Category is a class to define the group of places or connection on the digital map for LBS services. Category is used to help to develop the LBS indoor navigation system with different types of serives such as in education, department store. For example, the groups of a seraching category could be shop, immigraion agency, meeting room, and class room. The attrbitues of "Category" are category ID, and category name.

Route: Route is a class to define the way of a user to navigate from a starting location to the destination. The route class has "Location" and "Link". Link will be defiend next. In 
our ontology we define two types of routes for user indoor navigation: Emergency_Route, Normal_Route. These two types of routes are detemined by the status of the building situation. The atributes of the Route class is desigend using Route ID, Location ID, Node_ID and Distance.

Link: Link is a class to define the connection from a node to a different floor of the building vertically. Link has the atributes of Link ID and Link Type.

Some of the attributes used in our ontology are described in Table 1 . The deailed design of this ontology is descirbed in next section.

Table 1. Terminologies for Some Attributes in Ontology

\begin{tabular}{|l|l|l|}
\hline Terminology & \multicolumn{1}{|c|}{ Definition } & \multicolumn{1}{c|}{ Example } \\
\hline ID & $\begin{array}{l}\text { ID is a unique label for a Node, } \\
\text { Link, Route, or a Zone }\end{array}$ & $\begin{array}{l}\text { L51-Nd2_0001 (Level } \\
\text { 5, Node 2, 001) }\end{array}$ \\
\hline $\begin{array}{l}\text { Coordinate } \\
\text { system }\end{array}$ & $\begin{array}{l}\text { The coordinate system is defined } \\
\text { in 3 dimensions, two horizontal } \\
\text { and one vertical. }\end{array}$ & $\begin{array}{l}\text { (0,0,0) can be defined } \\
\text { at one entry location of } \\
\text { a building. }\end{array}$ \\
\hline Coordinate & (X, Y, Z) in Euclidean space & (23.45, 47.81, 4.00) \\
\hline Place_name & $\begin{array}{l}\text { Place name or all connection in } \\
\text { the building }\end{array}$ & Café, Room11.04 \\
\hline Level & $\begin{array}{l}\text { The floor number of a building } \\
\text { Zone zone is an area specified on the }\end{array}$ & $\begin{array}{l}\text { Zone51 (Zone 1 on } \\
\text { level 5) }\end{array}$ \\
\hline Category & $\begin{array}{l}\text { The category is the type of place } \\
\text { selected for display for users in } \\
\text { the mobile application. }\end{array}$ & $\begin{array}{l}\text { Shop, Service, Toilet, } \\
\text { etc. }\end{array}$ \\
\hline Status & $\begin{array}{l}\text { Node has status over time and } \\
\text { situation. }\end{array}$ & $\begin{array}{l}\text { On is working and Off } \\
\text { is not working. }\end{array}$ \\
\hline
\end{tabular}

\section{Detailed Design of the Ontology}

To design the ontology at detailed level, we design the nodes to present the physical concepts. Figure 2 illustrates the digital map in a building, and the nodes and links concepts from the ontology.

The major contribution of our ontology is a new node property. We designed three types of nodes: Location-Node to represent a physical indoor location in a building, UserMobile-Node to present the user dynamic location and Device Node to represent the device location. These nodes are designed as follows.
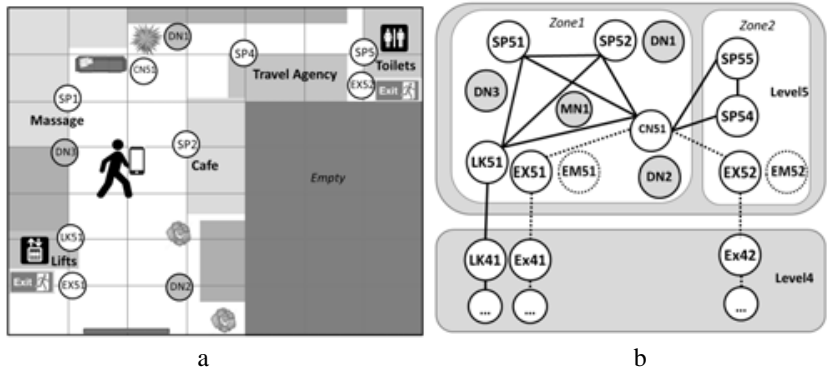

Fig. 2. (a) map and nodes (b) nodes and links

\section{Mobile Location Node:}

In our design, the location of a user carrying a smartphone will be assigned an active node or blue dot, called "UserMobile Node" (MN). This is a dynamic node. The attributes of $\mathrm{MN}$ will allow calculation of the current position.

\section{Device Location Node:}

We design Device Node (DN) for calculating the current user location. The devices are able to get measurements for location calculation.

\section{Place Location Node:}

We design each node such as shop (SP) to connect with others and use the connection (CN) for connecting to a zone area on the same floor. The building may have more than one level. The nodes should be able to link to other levels. Link is some way of going to other levels of the building. For example, as illustrated in Figure 2, we use the Link $(L K)$, such as LK5 on Level 5 of the building, to connect to LK4 on Level 4. There may be different choices for the route, such as using an elevator or escalator to connect to an another level. Using CN or LK can improve the processing efficiency. We designed a status concept for each node. The status of the node assists in the recommendation of the route for real-time indoor navigation.

The multi-level information nodes show how to connect all nodes together on the vertical layer information. We design a "Map Sheet" to include Links and Zones. Links and Zones are the connection point to connect to other levels and area. See Figure 3. A route array will be created when a user requests a way-finding search.

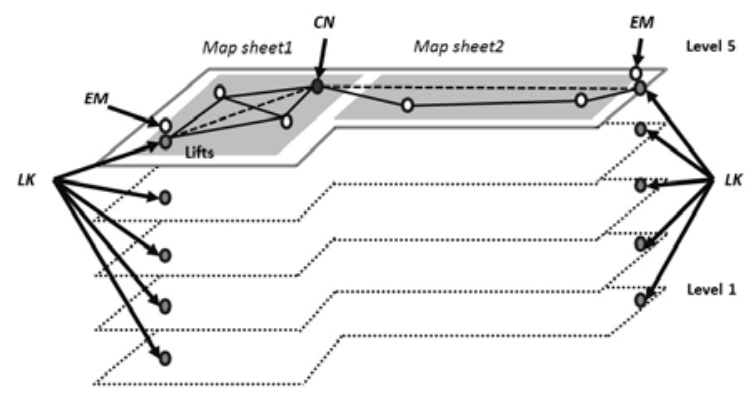

Fig. 3. Map sheet, and connection to different levels of the building

We designed "Zone" in the building digital map at all building levels. Map sheet builders can divide a floor to a number of zones for efficient management.

\section{APPLYING INDOOR NAVIGATION ONTOLOGY MODEL TO LBS}

Our ontology can be applied to indoor navigation for LBS using the flow from industry practice: Indoor Positioning /Geofencing / Way-Finding [7]:

\section{A. Indoor Positioning}

Figure 4 shows the concepts of indoor positioning using our ontology. A user can use the devices deployed at nearby Device Nodes (DL), and smartphone sensors at user's mobile location (ML) to get location measurements, and compute the user positioning information. Place Location Node (PL) I the destination for way-finding in later step. 


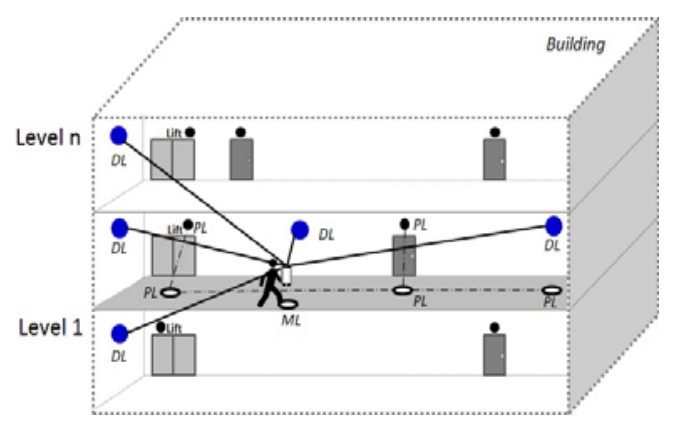

Fig. 4. Indoor positioning using Device Location and User Mobile Location

The status "On/Off” is desigend to assign to all nodes. The status will be applied in different situations of the building. If the status of one node is set to 'Off" in an emergency situation, the indoor positioning will use the nearby node which is set to 'On' status for an emergency.

\section{B. Geo-fencing}

Once the current user position is determined, the LBS navigation system will display the places $\left(\right.$ PLnode $\left._{\mathrm{i}}\right)$ within a designed zone area.

\section{Way-Finding}

The Route class in our ontology will be applied in wayfinding using route array design. Route_Array $\left(L K_{1}, S P_{1}, C N_{1} \ldots\right.$ $\left.L K_{i}, S P_{i}, C N_{i}\right)$ is an example to connect Link (LK) and Shops (SP) and Connections (CN).

For a high assurance LBS, we consider two types of routes: Regular Route and Emergency Route using the node status.

1) Regular Route: Most of the time, the building is in a usual status without any special situations such as on fire or the attack. The LBS map will display the regular route using the way-finding algorithm for selecting nodes which status are set to "On".

2) Emergency route: When the building is in an emergency situation, or some location nodes are in maintenance, these location nodes are set to "Off". We will find other nodes for calculating the current position from device nodes remains.

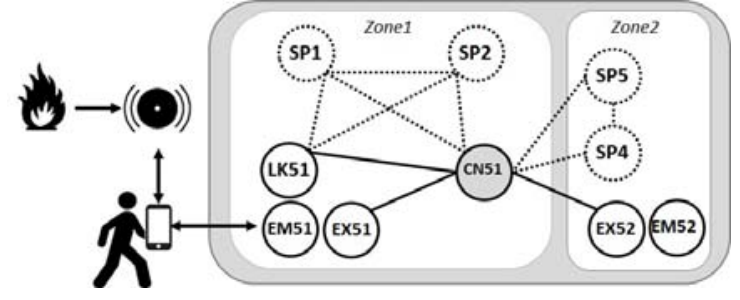

Fig. 6. Emergency nodes

Figure 6 shows the application when a fire alarm turns on, the associated sensor on a smartphone will analyse and response the status of location nodes. The status of some nodes will be changed to "Off" for updating the normal route. Emergency nodes will be changed to "On". The indoor navigation system is switched to emergency mode and display the emergency route to users.

\section{Conclusions And Future RESEARCH DiRECTION}

In this paper, we present an ontology approach for LBS indoor navigation. We designed a node concept to ontology model with three types of nodes: static location nodes, dynamic user-mobile node and static device nodes. The nodes are assigned a status for different building situation such as in emergency situation. The multi-level attributes are designed into the node. This ontology is able to support the emergency route selection. This reseach makes the designed LBS indoor navigation system more reliable.

In future research, we will work on using minimum devices to detect the current position inside the building. It will make the LBS inexpensive and highly accurate for indoor navigation. Moreover, the Ontology model may apply to communicate between users and rescuers in the emergencies. It would enable the rescuers to find the optimal route for victim searching.

\section{REFERENCES}

[1] Wang, H. Sensing Information Modelling for Smart City. in 2015 IEEE International Conference on Smart City/SocialCom/SustainCom (SmartCity). 2015: IEEE.

[2] Dehghanzadeh, R., K. Ansarian, and H. Aslani, Concentrations of carbon monoxide in indoor and outdoor air of residential buildings. Journal of Health, 2013. 3(4): p. 29-40.

[3] Bejuri, W.M.Y.W., et al., Emergency Rescue Localization (ERL) using GPS, Wireless LAN and Camera. International Journal of Software Engineering and Its Applications, 2015. 9(9): p. 217-232.

[4] Zipf, A. and M.M. Jöst, Location-based services, in Springer Handbook of Geographic Information. 2011, Springer. p. 417-421.

[5] Chung, J., et al. Indoor location sensing using geo-magnetism. in Proceedings of the 9th international conference on Mobile systems, applications, and services. 2011: ACM.

[6] Zàruba, G.V., et al., Indoor location tracking using RSSI readings from a single Wi-Fi access point. Wireless networks, 2007. 13(2): p. 221-235.

[7] Sterling, G., Magnetic positioning? the arrival of indoor gps. 2014, Opus Research Report.

[8] Newman, N., Apple iBeacon technology briefing. Journal of Direct, Data and Digital Marketing Practice, 2014. 15(3): p. 222-225.

[9] Maedche, A., Ontology learning for the semantic web. Vol. 665. 2012: Springer Science \& Business Media.

[10] Bechhofer, S., OWL: Web ontology language, in Encyclopedia of Database Systems. 2009, Springer. p. 2008-2009.

[11] Kessel, M., P. Ruppel, and F. Gschwandtner, BIGML: A location model with individual waypoint graphs for indoor location-based services. PIKPraxis der Informationsverarbeitung und Kommunikation, 2010. 33(4): p. 261-267.

[12] Kim, J.-S., S.-J. Yoo, and K.-J. Li. Integrating IndoorGML and CityGML for indoor space. in International Symposium on Web and Wireless Geographical Information Systems. 2014: Springer.

[13] Wang, X., et al., Indoor Semantic Location Models for Location-Based Services. International Journal of Smart Home, 2013. 7(4): p. 127-136.

[14] Tsetsos, V., et al., Semantically enriched navigation for indoor environments. International Journal of Web and Grid Services, 2006. 2(4): p. $453-478$

[15] Yim, J., J. Joo, and G. Lee, Petri net-based ontology analysis method for indoor location-based service system. International Journal of Advanced Science and Technology, 2012. 39: p. 75-92.

[16] Scholz, J. and S. Schabus. An indoor navigation ontology for production assets in a production environment. in International Conference on Geographic Information Science. 2014: Springer.

[17] Chen, R., et al., An indoor location system based on neural network and genetic algorithm. International Journal of Sensor Networks, 2015. 19(3-4): p. 204-216.

[18] Hofmann-Wellenhof, B., H. Lichtenegger, and J. Collins, Global positioning system: theory and practice. 2012: Springer Science \& Business Media. 